\title{
The Non-properness of the functor of $F$-trivial bundles
}

\author{
V.B. Mehta* and S. Subramanian ${ }^{\dagger}$
}

September 24, 2018

\begin{abstract}
We study the properness of the functor of $F$-trivial bundles by relating it to the base change question for the fundamental group scheme of Nori.
\end{abstract}

\section{Introduction}

Let $X$ be a non-singular projective variety over an algebraically closed field of arbitrary characteristic with a very ample line bundle $H$ on $X$. The notion of a coherent torsion-free sheaf being stable or semistable with respect to $H$ is now classical [15, 8]. In particular their moduli spaces with fixed Chern classes have been constructed [loc. cit]

In particular the property of the functor of semistable sheaves being proper is of crucial importance for $\operatorname{dim} X=1$, this was proved by Seshadri and then in general by Langton [9]. For chi-semistability, or GiesekeiMaruyama semistability, this was proved by Mehta-Ramanathan [10] and Maruyama. The properness of the semi-stable functor for $G$-bundles was also considered by Ramanathan for curves in characteristic zero, (see also BalajiSeshadri [2] and Faltings [4]) then by Balaji-Parameswaran [1] for curves in

*This paper was presented by the first author at a conference for Peter Russell at McGill University, Montreal in June 2009. He would like to thank the organizers D. Daigle, R. Ganong, J. Hurtubise, M. Koras and S. Lu for the invitation and hospitality.

$\dagger$ Vikram Mehta passed away on $4^{\text {th }}$ June, 2014. 
characteristic $p$ and then by Heinloth [7] and Gomez-Langer-Sols-Schmitt [5] for arbitrary varieties in characteristic $p$.

Denninger-Werner consider a slightly more general question [3]. Let $k$ be an algebraically closed field of characteristic $p$ and $W=W(k)$ the ring of Witt vectors over $k$, with function field $K$ and residue field $k$. Let $X \rightarrow \operatorname{Spec}(K)$ be a smooth, projective absolutely irreducible curve over $K$. Assume that $V$ is a semistable bundle of degree zero over $X$.

They ask the following question:

Does there exist a model $\bar{X}$ of $X$, and an extension $\bar{V}$ of $V$ to $\bar{X}$, such that for each irreducible component $Y_{i}$ of $\bar{X}_{k}$, the restriction of $V$ to the normalization of $\left(Y_{i}\right)_{\text {red }}$ is strongly semistable. Note that the original $V$ on $X_{K}$ may be considered strongly semistable as characteristic $K=0$. Such a bundle $V$ on $X_{K}$ is said to be have strong semistable reduction. Suppose there exists a finite morphism $f: Z \rightarrow X$ over $K$ and a model $\bar{Z}$ of $Z$ such that for every irreducible component $Z_{i}$ of $\bar{Z}_{k}$, the bundle $f^{*}(V)$ is strongly semistable on each normalization of $Z_{i_{\text {red }}}$. Then $V$ is said to have potentially strong semistable reduction. Note the analogies with the semistable reduction of vector bundles and principal bundles mentioned earlier.

For vector bundles with a strong semistable reduction, Denninger-Werner show that there are functorial isomorphisms of "parallel transport" along etale paths between the fibres of $V_{\bar{K}}$ on $X_{\bar{K}}$, where $\bar{K}$ is the algebraic closure of $K$. See also Hackstein [6] for a similar discussion on $G$-bundles.

In another direction, Madhav Nori had introduced the fundamental group scheme of a reduced projective scheme $X$ over $k$, denoted by $\Pi^{N}(X)$, [13, 14]. This is defined by assigning a Tannaka group to the Tannaka category of essentially finite vector bundles on $X$ [loc.cit]. In these papers, Nori had made 2 conjectures:

(1) If $X$ and $Y$ reduced, complete schemes over $k$, then $\Pi^{N}\left(X \times_{k} Y\right) \simeq$ $\Pi^{N}(X) \times_{k} \Pi^{N}(Y)$.

(2) If $l$ is an algebraically closed field extension of $k$, then the canonical map $\Pi^{N}\left(X_{l}\right) \rightarrow \Pi^{N}(X) \otimes_{k} l$ is an isomorphism.

The present authors had proved conjecture (1) in [11], using the the notion of an " $F$-trivial vector bundle". They had also introduced the local fundamental group-scheme of $X$ denoted by $\Pi^{\text {loc }}(X)$, using the Tannaka category of $F$-trivial bundles on $X$ [12]. In [loc. cit] they had also proved some necessary and sufficient conditions for the second conjecture of Nori to 
be valid. In an attempt to prove the second conjecture of Nori, they had formulated the following question:

Question 1: Let $X$ be a smooth projective curve, and let $S_{0}$ be a smooth affine curve with a smooth completion $S$. Let $V_{0}$ be a vector bundle on $X \times S_{0}$ such that for every $s$ in $S_{0}, V \mid X \times\{s\}$ is $F$ - trivial on $X$. Then can $V_{0}$ be extended to a vector bundle $V$ on $X \times S$ such that for every $s$ in $S, V \mid X \times\{s\}$ is $F$-trivial on $X$ ?

This may be thought of as a properness theorem for $F$-trivial vector bundles on $X$.

We may also consider the following question:

Question 2: Let $X$ be a nonsingular projective curve on $k$ and let $T_{0}$, be any smooth affine curve. Let $V$ be a vector bundle on $X \times T_{0}$ such that

1. for all $t \in T_{0}$, the bundle $V \mid X \times\{t\}$ is $F$-trivial on $X$.

2. for all $t$ in a non-empty open subset $U$ of $T_{0}$, the bundle $V \mid X \times\{t\}$ is stable on $X$ (and also $F$-trivial on $X \times\{t\}$ ) for all $t \in U$.

Then is the classifying map $c: T_{0} \rightarrow U_{X}(r, 0)$ constant?

Here $U_{X}(r, 0)$ denotes the moduli spaces of rank $r$ and degree 0 semistable vector bundles on $X$. Note that any $F$-trivial vector bundle on $X$ is strongly semistable of degree 0 [12].

In this paper we prove that an affirmative answer to Question 1 leads to an affirmative answer to Question 2. It is important to note here that an affirmative answer to Question 2 would prove Nori's second conjecture. In fact, Nori's second conjecture is equivalent to Question 2. [Section 3].

But Christian Pauly has given a counter-example to Nori's second conjecture [16]. He constructs a nonconstant family of stable, $F$-trivial vector bundles, which is not constant. Therefore, Question 2, is false, hence Question 1 has also a negative answer.

This also shows that in the equicharacteristic $p$ case, the question of Denninger-Werner also has a negative answer when one fixes a smooth and projective model for $X$, that is when the special fibre is a smooth, projective curve. For all the results used here, about stability, semistability, $F$-trivial bundles and the precise statements of Nori's 2 conjectures, we refer to [15, 12, 


\section{Formulation and some Lemmas}

Here we collect some basic facts about $F$-trivial bundles. Throughout we work over an algebraically closed field $k$ of characteristic $p$. If $X$ is a scheme (reduced) of finite type over $k$, we denote by $F$, the Frobenius map $X \rightarrow X$. As $k$ is assumed to be perfect, we do not distinguish between the geometric Frobenius and the absolute Frobenius. We have

Definition: A vector bundle $V$ on $X$ is said to be $F$-trivial if $F^{*}(V) \simeq \mathcal{O}_{X}^{\oplus r}$, where $r=\operatorname{rank} V$ [11].

Remark 2.1. For any integer $n>1$, we could define on $F^{n}$-trivial bundles on $X$ as a bundle $V$ on $X$ such that $F^{n *}(V) \simeq \mathcal{O}_{X}^{\oplus r}, r=$ rank $V$. But for ease of notation we assume $n=1$. See however, the remarks at the end of section 3. We rephrase Questions 1 and 2 as Statements:

Statement 1 Let $X$ be a nonsingular projective curve and $S_{0}$ a smooth affine curve, with smooth completion $S$ (everything is defined over $k$ ). Let $V_{0}$ be a vector bundle on $X \times S_{0}$ such that for all $s \in S_{0}, V_{s}:=V \mid X \times\{s\}$ is $F$-trivial on $X$. Then $V_{0}$ can be extended to a bundle $V$ on $X \times S$ such that for all $s \in S, V_{s}:=V \mid X \times\{s\}$ is $F$-trivial on $X$.

Note that if such an extension exists, then it is unique, as $F$-trivial bundles are semistable, and by Langton's Theorem. Using Statement 1, we shall prove

Statement 2 Let $X$ be a nonsingular projective curve and $T_{0}$ a smooth affine curve. Assume that there exists a vector bundle $V_{0}$ on $X \times T_{0}$ such that

1) for all $t \in T_{0}, V_{t}:=V_{0} \mid X \times\{t\}$ is $F$-trivial on $X$.

2) for all $t$ in a non-empty open subset $U$ of $T_{0}, V_{t}$ is stable on $X$. Then the family $V_{0}$ is constant, that is the classifying map $c_{T_{0}}: T_{0} \rightarrow U_{X}(r, 0)$ is constant, where $U_{X}(r, 0)$ is the moduli space of rank $\mathrm{r}$ and degree 0 semistable bundles on $X$.

Assuming Statement 1, we prove Statement 2 in a sequence of Lemmas:

Let $V_{0}$ on $X \times T_{0}$ be as in Statement 2. By Statement $1, V_{0}$ can be extended to a vector bundle $V$ on $X \times T$, where $T$ is a smooth completion of $T_{0}$, such that for all $t \in T, V_{t}:=V \mid X \times\{t\}$ is $F$-trivial on $X$. Then we have 
Lemma 2.2. For any $x \in X$, consider the bundle $V_{x}:=V \mid\{x\} \times T$ on $T$. Then $\left\{V_{x}\right\}, x \in X$, considered as a family of bundles on $T$ parameterized by $X$, is constant. That is, $V_{x} \simeq V_{y}$ as bundles on $T$, for any pair of points $x, y$ in $X$.

Proof. Let $F_{X}: X \rightarrow X$ be the Frobenius map of $X$. Consider $\left(F_{X} \times\right.$ $\left.\operatorname{id}_{T}\right)^{*}(V)$ on $X \times T$. As $F_{X}^{*}\left(V_{t}\right)$ is trivial on $X$, for any $t \in T$, we have $\left(F \times \mathrm{id}_{T}\right)^{*}(V) \simeq p_{2}^{*}(W)$ for some vector bundle on $W$ on $T$, by semicontinuity. Hence $\left(F_{X} \times \operatorname{id}_{T}\right)^{*}(V) \mid\{x\} \times T \simeq W$ on $T$. But clearly, $V \mid\{x\} \times T$ and $\left(F_{X} \times \operatorname{id}_{T}\right)^{*}(V) \mid\{x\} \times T$ are isomorphic as bundles on $T$, as $F_{X}: X \rightarrow X$ is surjective. Hence the Lemma.

In what follows we shall call $W$ the parameter bundle on $T$.

Lemma 2.3. Without loss of generality, $W$ may be assumed to have degree 0 on $T$.

Proof. If degree $W=0$, we are through. Otherwise, let degree $W=d$ and rank $W=r=\operatorname{rank} V$. We can certainly find a map. $f: Z \rightarrow T$, where $Z$ is a smooth curve and a line bundle $L$ on $Z$ such that $r \operatorname{deg} L+\operatorname{deg} f^{*}(W)=0$ Consider the family $\left(\operatorname{id}_{X} \times f\right)^{*}(V) \otimes p^{*} L^{-1}$ on $X \times Z$. The parameter bundle for this family is clearly $f^{*}(W) \otimes L^{-1}$, which has degree 0 on $Z$. The new family on $X \times Z$ has the same properties as $V$ on $X \times T$. And if $c_{Z}: Z \rightarrow$ $U_{X}(r, 0)$ is constant, so is the map $c_{T}: T \rightarrow U_{X}(r, 0)$ where $c_{T}$ and $c_{Z}$ are the classifying map for $V_{0}$ on $X \times T$ and $\left(\operatorname{id}_{X} \times f\right)^{*}(V) \otimes p_{2}^{*} L^{-1}$ on $X \times Z$ respectively. So we may assume that the degree of the parameter bundle $W=0$.

Now we make an assumption that will be removed in Section 3 $(*)$ : The ground field $k$ is the algebraic closure of $F_{p}$, that is $k \simeq \bar{F}_{p}$.

With this assumption we have

Lemma 2.4. $W$ is strongly semistable on $T$.

Proof. If not, $F^{m^{*}}(W)$ has a strong Harder-Narasimhan filtration: $0=$ $A_{0} \subset A_{1} \subset \cdots \subset A_{n}=W$ with each $A_{i} / A_{i-1}$ strongly semistable and $\mu\left(A_{i} / A_{i-1}\right)>\mu\left(A_{j} / A_{j-1}\right)$ if $i<j$. Denote each $A_{i} / A_{i-1}$ by $B_{i}$. Just as in Lemma 2.3, $\exists f: Z_{i} \rightarrow T$ such that $f^{*}\left(B_{i}\right)$ has degree 0 , with $Z_{i}$ a smooth curve. But $f^{*}\left(B_{i}\right)$ is still strongly semistable on $Z_{i}$. This implies that 
$f^{*}\left(B_{i}\right)$ is essentially finite on $Z$ [18]. This further implies that there exists a smooth projective curve $S$ and a map $g: S \rightarrow Z$ with $g^{*}\left(f^{*} B_{i}\right)$ trivial on $S$. Trivializing $B_{1}, \ldots, B_{n}$ this way, we get finally a smooth projective curve $R$ and a map $h: R \rightarrow T$ such that $h^{*}(W)$ is a direct sum,

$$
h^{*}(W) \simeq L_{1}^{\oplus r_{1}} \oplus L_{2}^{\oplus r_{2}} \cdots \oplus L_{s}^{\oplus r_{s}}
$$

for some line bundles $L_{i}, 1 \leq i \leq s$ on $R$ with $\operatorname{deg} L_{1}>\operatorname{deg} L_{2} \ldots>\operatorname{deg} L_{s}$ and some positive integers $r_{i}$, with $\sum r_{i}=r$. Consider the family $\left(\operatorname{id}_{X} \times\right.$ $h)^{*}(V)$ on $X \times R$. If the classifying map $c_{R}: R \rightarrow U_{X}(r, 0)$ is constant, then the classifying map $c_{T}: T \rightarrow U_{X}(r, 0)$ is also constant. Hence we may assume, that on $T$ itself the parameter bundle is a direct sum, $W \simeq L_{1}^{\oplus r_{1}} \oplus \cdots \oplus L_{s}^{\oplus r_{s}}$ with each $L_{i} \in \operatorname{Pic}(T)$.

Now consider $V$ as a family of vector bundles on $T$ parametrized by $X$. Consider the relative Harder-Narasimhan filtration of $V$ on $T$, relative to $X$. By semi-continuity $\exists$ vector bundles $V_{1}, \ldots, V_{s}$ on $X$ of ranks $r_{1}, \ldots, r_{s}$, and a filtration on $X \times T$

$$
0=W_{0} \subset W_{1} \cdots \subset W_{s}=V
$$

such that each $W_{i} / W_{i-1} \simeq p_{1}^{*}\left(V_{i}\right) \otimes p_{2}^{*}\left(L_{i}\right)$ Now consider the family $\left(F_{X} \times\right.$ $\left.\mathrm{id}_{T}\right)^{*}(V)$ on $X \times T$. This bundle is isomorphic to $p_{2}^{*}(W)$ by Lemma 2.2. So

$$
p_{2}^{*}(W) \simeq p_{2}^{*} L_{1}^{\oplus r_{1}} \oplus \cdots \oplus p_{2}^{*} L_{s}^{\oplus r_{s}}
$$

Apply $\left(F_{x} \times \mathrm{id}_{T}\right)^{*}$ to filtration $(1)$, we get

$$
0 \subset F_{X}^{*}\left(W_{1}\right) \ldots \subset F_{X}^{*}\left(W_{s}\right)=F_{X}^{*}(V)
$$

with each $F_{X}^{*}\left(W_{i}\right) / F_{X}^{*}\left(W_{i-1}\right) \simeq p_{1}^{*}\left(F_{X}^{*} V_{i}\right) \otimes p_{2}^{*}\left(L_{i}\right)$.

Compare filtration (2) and (3):

It is clear that $p_{2}^{*} L_{1}^{\oplus r_{1}}$ has no maps to $p_{1}^{*}\left(F_{X}^{*} V_{i}\right) \otimes p_{2}^{*}\left(L_{i}\right)$ for $i>1$. as degree $L_{1}>$ degree $L_{2} \ldots>$ degree $L_{s}$.

Hence $p_{2}^{*} L_{1}^{\oplus r_{1}}$ injects into $p_{1}^{*}\left(F_{X}^{*} V_{1}\right) \otimes p_{2}^{*}\left(L_{1}\right)$ on $X \times T$. Tensoring by $p_{2}^{*}\left(L_{1}^{-1}\right)$, we get an injection of the trivial bundle of rank $r$, into $p_{1}^{*}\left(F_{X}^{*} V_{1}\right)$ on $X \times T$. Hence on $X$, we get an injection of the trivial bundle into $F_{X}^{*} V_{1}$. This implies that degree $F_{X}^{*} V_{1} \geq 0$ hence degree $V_{1} \geq 0$. Choose any closed point $t \in T$ and restrict filtration (1) to $X \times\{t\}$ we get an injection of $V_{1}$ inside $V_{t}$. But degree $V_{1}=0$ and $V_{t}$ is stable for a a general $t \in T$, which is a contradiction. Hence $W$ is strongly semistable on $T$. 
Theorem 2.5. The classifying map $c_{T}: T \rightarrow U_{X}(r, 0)$ is constant.

Proof. Consider the sequence of bundles on $T$, given by $F^{n^{*}}(W), n=$ $1,2, \ldots$. They are all semistable of degree 0 . As we are working over $k=\bar{F}_{p}$, we must have $F^{n^{*}}(W) \simeq F^{m^{*}}(W)$ for some positive integers $m, n$ with $m \neq n$. By [18, $W$ is essentially finite on $T$, that is there exists a smooth projective curve $Z$ and a map $h: Z \rightarrow T$ such that $h^{*}(W)$ is trivial. Consider the family $\left(\operatorname{id}_{X} \times h\right)^{*}(V)$ on $X \times Z$. This has parameter bundle $h^{*}(W)$, which is trivial. So $\left(\operatorname{id}_{X} \times h\right)^{*}(V) \simeq p_{1}^{*}(A)$ for some vector bundle $A$ on $X$. But then the classifying map $c_{Z}: Z \rightarrow U_{X}(r, 0)$ is constant, so the classifying $\operatorname{map} c_{T}: T \rightarrow U_{X}(r, 0)$ is also constant.

\section{Main result}

Now we remove the assumption $(*)$ in Section 2 . So let $k$ be any algebraically closed field of characteristics $p$ and $V_{0}$ on $X \times T_{0}$ be as in Statement 2. By Statement $1, V_{0}$ extends to a family $V$ of $F$-trivial bundles on $X$, parameterized by $T$, where $T$ is a smooth completion of $T_{0}$ Then we have

Theorem 3.1. The classifying $\operatorname{map}_{T}: T \rightarrow U_{X}(r, 0)$ is constant.

Proof. $W$ is defined by the isomorphism $\left(F_{X} \times \mathrm{id}_{T}\right)^{*}(V) \simeq p_{2}^{*}(W)$ on $X \times T$. We may assume that there exists an algebra $R$, finitely generated over $F_{p}$ such that for $V$ on $X \times T$, there exist models:

$$
\begin{array}{llll}
\text { 1) } & X_{R} \rightarrow R & \text { for } & X \\
\text { 2) } & T_{R} \rightarrow R & \text { for } & T \\
\text { 3) } & V_{R} \rightarrow X_{R} & \text { for } & V \\
\text { 4) } & W_{R} \rightarrow T_{R} \text { for } & W
\end{array}
$$

We may also assume that there exist open nonempty subsets $O_{1}$ and $O_{2}$ of $\operatorname{Spec}(R)$ such that 1) and 2) below are satisfied:

1) For every geometric point $\operatorname{Spec}(\omega) \rightarrow O_{1}$ with image a closed point $m$ of $O_{1}$, the bundle $V_{\omega}$ is a family of $F$-trivial bundles on $X_{\omega}$, parameterized by $T_{\omega}$. This is seen as follows : the bundle $W$ on $T$ has a model $W_{R} \rightarrow T_{R}$ and the isomorphism $\left(F_{X} \times \operatorname{id}_{T}\right)^{*}(V) \simeq p_{2}^{*}(W)$ can be spread out over $O_{1}$. This proves that for every closed point $t$ of $T_{\omega}$, the bundle $V_{t}$ is $F$-trivial on $X_{\omega} \times\{t\}$. 
2)We may also assume that there exists a nonempty open subset $\mathrm{O}_{2}$ of $\operatorname{Spec} R$ such that for every geometric point $\operatorname{Spec}(\omega) \rightarrow \mathrm{O}_{2}$ with image a closed point $m$ of $\mathrm{O}_{2}$, the family $V_{\omega}$ on $X_{\omega} \times T_{\omega}$ is generically stable. This is proved as follows:

Let $U_{X_{R}}(r .0) \rightarrow \operatorname{Spec}(R)$ be the relative moduli space of semistable bundles of rank $r$ and degree 0 on the fibers of $X_{R} \rightarrow R$ [8]. Let $U_{X_{R}}^{s}$ be the open submoduli space of stable bundles. If $K$ is the quotient field of $R$, then we have assumed that the image of $c_{K}: T_{K} \rightarrow U_{X_{K}}$ intersects $U_{X_{K}}^{s}$. So there exists a nonempty open subset $O_{2}$ of $\operatorname{Spec}(R)$ such that $c_{m}: T_{m} \rightarrow U_{X_{m}}$ intersects $U_{X_{m}}^{s}$ for all closed points $m$ in $O_{2}$. So we may assume, without loss of generality, that $O_{1}=O_{2}=\operatorname{Spec} R$. For any closed point $m$ in $\operatorname{Spec} R$ we know that $c_{m}: T_{m} \rightarrow U_{X_{m}}$ is a constant map. It easily follows that $c_{K}: T_{K} \rightarrow U_{X_{K}}$ is a constant map, thus finishing the proof that Statement 1 implies Statement 2 over arbitrary algebraically closed fields in characteristic $p$.

Remark 3.2. For any integer $n>1$, we may define an $F^{n}$-trivial bundle $V$ on $X$ : we ask that $F^{n^{*}}(V)=\mathcal{O}_{X}^{\oplus r}, r=\operatorname{rank} V$. It is trivial to check that the proof that Statement 1 implies Statement 2 goes through without any changes.

Remark 3.3. But Statement 2 is false!! More precisely, Christian Pauly [16] has produced a non-constant family of stable bundles, trivialized by $F^{4}$, the fourth power of Frobenius on a curve of genus 2 in characteristic 2 . He has done this by a careful study of the Verschiebung map $V: U_{X}(r, 0) \rightarrow U_{X}(r, 0)$, induced by the Frobenius map $F: X \rightarrow X$. This also shows that the answer to the question of Denninger-Werner is also negative, if one works over a fixed smooth model of $X$. In fact the second conjecture of Nori implies Statement 1. We provide a sketch proof in the following:

Theorem 3.4. The second conjecture of Nori implies the properness of the functor of F-trivial bundles (i.e., Statement 1).

Proof. Let $V_{0}$ over $X \times T_{0}$ be as in Statement 1, and let $T$ be a smooth completion of $T_{0}$. We may assume, without loss of generality, that $T-T_{0}$ is one point, say $\infty$. We first extend $V_{0}$ to a family of semistable bundles on $X \times T$, that is, $V_{\infty}$ is semistable. Note that $V_{\infty}$ is not unique, but $\operatorname{gr}\left(V_{\infty}\right)$ is unique. If $V_{t}$ is stable for some point $t \in T_{0}$, then $V_{t}$ is stable for all $t$ in $U$, where $U$ is open in $T_{0}$. By [12], we know that the set of isomorphism classes 
$V_{t}$ is finite. This implies that the map $c_{U}: U \rightarrow U_{X}(r, 0)$ is constant and $c_{U}(t)$ is a fixed bundle $V$ in $U_{X}^{s}(r, 0)$ for all $t$ in $U$. As $T$ is connected, we must have $c_{U}(t)=V$ in $U_{X}^{s}(r, 0)$ for all $t$ in $T$, in particular $V_{\infty}$ is $F$-trivial on $X$. Assume that $V_{t}$ is strictly semistable for all $t \in T_{0}$. Let $\left[A_{t, i}\right]$ be the set of stable components of $V_{t}, t$ in $T_{0}$, and $1 \leq i<r=\operatorname{rank} V$. Again by [12], the set of isomorphism classes of $\left\{A_{t, i}\right\}$ is finite. But this implies that the map $c_{T_{0}} \rightarrow U_{X}(r, 0)$ has a finite image, hence constant as $T_{0}$ is connected. So again, we get $\operatorname{gr}\left(V_{\infty}\right)=\operatorname{gr}\left(V_{t}\right)$ for all $t$ in $T$.

But this implies that $V_{\infty}$ is strongly semistable. (All the Frobenius pull back are semistable of degree 0 ). Now consider the family $F_{X}^{*}\left(V_{t}\right), t \in T$. This a family of trivial bundles converging to a semistable bundle $F^{*}\left(V_{\infty}\right)$. So $F_{X}^{*}\left(V_{\infty}\right)$ is also trivial, so $V_{\infty}$ is $F$-trivial.

Remark 3.5. To complete the circle of ideas, we note that Statement 2 implies the second conjecture of Nori. We prove this now.

Theorem 3.6. The validity of Statement 2 implies the second conjecture of Nori.

Proof. Let $k \subset K$ be algebraically closed fields of characteristic $p$ and let $V$ be a stable $F$-trivial bundle on $X_{K}:=X \otimes_{k} K$. We check the criterion in [12]. So we have to show that $V$ is defined over $k$, that is over $X$. We can find $R$, a finitely generated algebra with quotient field $L \subset K$ such that $X_{K}$ has a model $X_{R} \rightarrow R$ and $V$ has a model $V_{R} \rightarrow X_{R}$. By cutting down $\operatorname{Spec}(R)$ suitably, we may assume that for all geometric points $\operatorname{Spec}(\omega) \rightarrow$ $\operatorname{Spec}(R)$, the bundles $V_{\omega}$ are stable and $F$ trivial on $X_{\omega}$. For any curve $T_{0}$ in $\operatorname{Spec}(R)$ the family $V_{R} \mid X_{R} \times_{R} T_{0}$ is constant by assumption. So the map $c_{R}: \operatorname{Spec}(R) \rightarrow U_{X}(r, 0)$ is constant, where $r=\operatorname{rank} V$. But this means that $V$ is defined over $k$, that is, $V$ comes from $X$.

Remark 3.7. It maybe true that a generalized version of the properness theorem is true for the Nori fundamental group scheme. This would also imply the generalized conjecture of Denninger-Werner. We hope to return to these questions in the future.

\section{References}

[1] V. Balaji and A.J Parameswaran, Semistable Principal Bundles II Positive characteristics Transform Groups 8 (2003), no. 1, 3-36. 
[2] V. Balaji and C.S. Seshadri, Semistable Principal Bundles I. characteristic zero, J. Algebra 258 (2002), no. 1, 321-347.

[3] Denninger, C. and A. Werner, Vector bundles on $p$-adic curves and parallel transport, Ann. Sci. Ecole Norm. Sup. (4) 38(2005), no. 4, 553-597.

[4] Faltings, G. Stable G-bundles and projective connections, J. Algebraic Geom. 2 (1993), no. 3, 507-568.

[5] Gomez T; Langer, A; Schmitt, A.H.W.; Sols, I; Moduli spaces for principal bundles in arbitrary characteristic, Ad. Math. 219 (2008), no. 4, 1177-1245.

[6] Hackstein, U., Principal bundles on p-adic curves and parallel transport, Int. Math. Res. Not. IMRN 2008,

[7] Heinloth, J., Semistable reduction of $G$-bundles on curves, J. Algebraic Geom. 17(2008), no.1, 167-183.

[8] Langer A., Moduli Spaces of sheaves and principal G-bundles, Alg. geometry-Seattle 2005 Part I, 273-308, Proc. Symp. Pure Math., 80, part I, AMS, Providence RI, 2009.

[9] Langton, S.G.Valuative criteria for families of vector bundles on algebraic varieties Ann. of Math. (2) , 101 (1975), 88-220.

[10] Mehta, V.B. and Ramanathan, A., An analogue of Langton's theorem on valuative criteria for vector bundles, Proc. Roy. Soc. Edinburgh Sect. A 96 (1984), no. 1-2, 39-45.

[11] Mehta, V.B and Subramanian, S; On the fundamental group scheme. Invent. Math. 148 (2002), no. 1, 143-150.

[12] Mehta, V.B., and Subramanian , S; Some remarks on the local fundamental group scheme, Proc. Indian acad Sci. (math. Sci.) Vol. 118, No.2, May 2008, 207-211.

[13] Nori , M.V., the fundamental group scheme, Proc. Indian Acad. Sci. Math. Sci. 91 (1982), no. 2, 73-122.

[14] Nori. M.V., On the representations of the fundamental group, Compositio Math. 33(1976), no. 1. 29-41. 
[15] Newstead, P.E., Introduction to moduli problems and orbit spaces. TIFR Lectures on mathematics and physics, 51, Narosa Publishing House, New Delhi , 1978.

[16] Pauly, C., A smooth counter example to Nori's conjecture on the fundamental group scheme, Proc. Amer. Math. Soc. 135 (2007), no. 9, 27072711 .

[17] Ramanathan, A, Moduli for principal bundles over algebraic curves I and II Proc. Indian Acad, Sci. Math. 106(1996), no.3, 301-328 and no. 4, 421-449.

[18] Subramanian, S, Strongly semistable bundles on a curve over a finite field, Arch. Math. (Basel) 89 (2007), no.1, 68-72.

S.Subramanian

School of Mathematics

TIFR,Navy Nagar

Mumbai 400005

email:subramnn@math.tifr.res.in 